\title{
Poor growth in school entrants as an index of organic disease: the Wessex growth study
}

\author{
L D Voss, J Mulligan, P R Betts, T J Wilkin
}

\section{Abstract}

Objective-To establish whether poor height or height velocity, assessed during the year of school entry, might identify children with previously undiagnosed organic disease.

Design-Observation of a total population and their case controls.

Setting-Community base.

Subjects-All 14346 children in two health districts entering school during two consecutive years were screened for height by school nurses, and those whose height lay below the 3 rd centile according to Tanner and Whitehouse standards $(n=180)$ were identified. After excluding 32 with known organic disease, five from ethnic minorities, and three who refused to take part, the remaining 140 short normal children were matched with 140 age and sex matched controls of average height (10th-90th centile) and their height velocities over 12 months measured.

Main outcome measures-Height, height velocity, previously diagnosed organic disease, and organic disease diagnosed as a result of blood tests and specialist examination.

Results-Twenty five of the 180 short children $(14 \%)$ were already known to have chronic organic disease which could explain their poor growth. Blood tests and specialist examination revealed a further seven with organic disease, which was acquired rather than congenital in three, and a second cause of short stature in one with known organic disease. These eight conditions had been missed at the school entry medical examination. The shorter the child, the more likely an underlying organic disorder, with seven of the 12 children whose heights were more than 3 standard deviations below the mean having some organic disease. Height velocity measured over 12 months, however, did not distinguish short normal children from those with disease or from their matched controls.

Conclusions-Height, but not height velocity, is a useful index for identifying unrecognised organic disease at school entry. The shorter the stature the greater the prevalence of organic disease. The frequency of newly diagnosed remediable disease in this study (1 in 3-4000) is similar to that of neonatal hypothyroidism, which is routinely screened for. Routine investigation of all very short school entrants is recommended.

\section{Introduction}

Screening programmes aim at early identification of otherwise undetected disease.' The screening test may not itself be diagnostic but serve to identify those at risk so that they can be referred for further investigation. Growth is a sensitive indicator of a child's wellbeing, ${ }^{2}$ and the early identification of poor growth may provide a simple screening test for underlying unrecognised disease.

The value of medical examinations on school entry has recently been questioned, ${ }^{34}$ although data on the effectiveness of growth screening for unidentified disease are few. Some years ago Lacey and Parkin reviewed 98 ten year olds in Newcastle on Tyne whose heights lay below the 3 rd centile. ${ }^{5}$ They found 16 with organic disease, but only two with previously unidentified disease-one with a chromosomal abnormality, the other with a possible partial growth hormone deficiency. A further cohort of 32 very short children (heights more than 3 standard deviations below the mean) was analysed, and half were found to have organic disease. The authors concluded that unless there were clear symptoms or signs of disease, investigation of short children should be confined to those with heights below 3 standard deviations below the mean, or a slow growth rate. Vimpani et al examined 449 children with heights more than 2.5 standard deviations below the mean and they found $108(24 \%)$ to have an organic basis for their short stature, but only two who were previously unidentified. ${ }^{\circ}$ Reviewing the evidence, Cross concluded that there were insufficient data to support school based screening for growth problems. ${ }^{7} \mathrm{He}$ suggested that a slow rate of growth would be more likely to indicate some pathological cause but pointed out that there had been no study to establish the prevalence of low velocity.

Data from the Wessex growth study have allowed us to re-examine two questions: firstly, whether school based screening for short stature would successfully identify children with underlying organic disease, and, secondly, to establish whether short stature alone, or a poor rate of growth, would be the more effective screening tool.

\section{Subjects and methods}

All 5 year olds entering school between 1985 and 1987 in two adjacent health districts in Wessex were measured by school nurses. ${ }^{*}$ All the short children (below the 3rd centile for height according to Tanner and Whitehouse standards") were then invited to undergo a blood test (for haemoglobin concentration, full blood count, thyroid function, and urea and electrolyte, serum calcium, phosphate, and alkaline phosphatase concentrations), a urinary growth hormone measurement, and hand and wrist radiographs to measure bone age. Examination by a paediatric specialist followed where results were abnormal.

After all children with organic disease (32), those from ethnic minorities for whom Tanner and Whitehouse standards may not be appropriate (5), and those who refused to take further part (3) were excluded, the remaining 140 short normal children were matched for age, sex, and school class with 140 children of normal height (10th-90th centile). The heights of all these 
TABLE 1-Comparison of the heights and height velocities in the two groups of short children and their controls

\begin{tabular}{lccc}
\hline & Controls & Short normal & $\begin{array}{c}\text { Short with } \\
\text { organic disease }\end{array}$ \\
\hline Median height SD score & $+0 \cdot 13^{\star}(n=140)$ & $-2 \cdot 18+(n=140)$ & $-2 \cdot 60(n=29)$ \\
Mean velocity SD score (SD) & $+0 \cdot 30(0 \cdot 90)^{\star}(n=134)$ & $-0 \cdot 54(0 \cdot 83)+(n=137)$ & $-0 \cdot 43(1 \cdot 00)(n=20)$ \\
\hline
\end{tabular}

${ }^{\star}$ Height and 12 month velocity of controls significantly greater than ch short normals and those with organic disease $(\mathrm{p}<0.01)$. the Height of short normal children significantly greater than those with organic disease $(p<0.01)$ but 12 month velocity not significant different $(p=0.67)$.

TABLE II-Relation between height and the frequency of organic disease thought to account for it

\begin{tabular}{lrr}
\hline Height & No & $\begin{array}{c}\text { No (\%) with } \\
\text { organic } \\
\text { disease }\end{array}$ \\
\hline$<-2.0$ SD & 170 & $24(14)$ \\
$<-2.5$ SD & 55 & $17(31)$ \\
$<-3.0$ SD & 12 & $7(58)$ \\
\hline
\end{tabular}

All cases of known disease ( $n=$ 25) and all cases of new disease identified among 180 in children $<3$ rd height centile at school entry, 1985/7, in Winchester and Southampton health districts

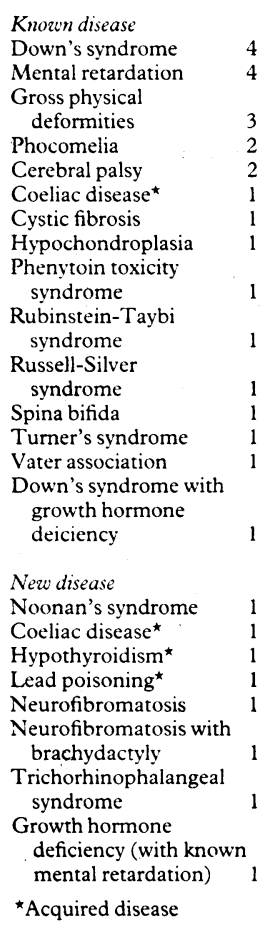
single auxologist (LDV) initially and again 12 months later, using a Holtain electronic stadiometer. The standard deviation for a single height measurement was $0.25 \mathrm{~cm} .{ }^{10}$ All growth data were referred to Tanner and Whitehouse standards, ${ }^{9}$ and heights and velocities were converted to $\mathrm{SD}$ scores to allow comparison of data in children of slightly different ages. SD score $=(\mathrm{x}-\overline{\mathrm{x}}) /(\mathrm{SD})$, where $\mathrm{x}=$ measurement and $\overline{\mathrm{x}}$ and $\mathrm{SD}=$ the mean and $\mathrm{SD}$ appropriate for age and sex.

Statistical analysis-Comparisons between groups were made using the Mann-Whitney test for height (non-normal distribution among short children) and Student's $t$ test for velocity, whose distribution was close to normal (fig 1). Data are expressed as means (SD).

\section{Results}

Screening procedure-A total of 14346 children were screened, and 180 children ( 97 boys and 83 girls) found to lie below the 3 rd centile for height. This figure represented only $1.25 \%$ of the school entry population instead of the expected $3 \%$, but was not surprising in view of the continuing secular trend. ${ }^{81112}$ Of those identified, $25(14 \%)$ were already known to have chronic organic disease which might have explained their short stature. Three of the 25 were so severely physically deformed as to make accurate height measurement difficult, and they were not further considered. Also excluded from further study was a child with Down's syndrome receiving growth hormone treatment. The routine school entry medical examination of school entrants had not revealed further organic disease to account for short stature in the remaining children.

Causes of organic disease-Eighty four per cent of the apparently normal short children underwent a blood test (128/152). This, together with specialist examination, revealed a further seven children with organic disease. Another child with known mental retardation was found also to be growth hormone deficient. There were, therefore, 32 children in total with organic disease $(18 \%)$ identified among 180 children whose height on screening at school entry was on or below the 3 rd centile.

The box lists both the diseases already detected between birth and 5 years and those identified by further investigations and examination. There was a higher prevalence of acquired disease in the latter group (3/7) than in the former (1/25).

Heights of children-The median height of the children with organic disease whose height was accurately measured (29) was significantly less than that of the remaining (normal) short children who agreed to take part in the study $(p<0.01)$. The data are summarised in table I. Furthermore, the shorter the child the more likely the presence of an underlying organic disorder (table II). Thus, the proportion of children with organic disease rose from $14 \%$ of all children whose height was $2 \mathrm{SD}$ or more to $58 \%$ of those whose height was 3 SD or more below the mean. The heights of 23 short asthmatic children and 32 judged to have psychosocial deprivation (median $-2 \cdot 21$ and $-2 \cdot 26$ respectively) were not statistically different from those of the remaining normal short children (median $-2 \cdot 15)$ and were therefore included in the data.

Height velocities of children-Three short and six children and their controls were then measured by a control children left the area and were not remeasured. The data summarised in table I compare the mean height velocities obtained for 20 short children with organic disease, 137 normal short children, and 134 of their controls after the first 12 months. The figure shows the velocity SD score. Velocity data for four children with organic disease were not calculated because a sufficiently accurate height measurement was impossible to obtain; one other refused follow up; and a further four were receiving treatment: the child with known coeliac disease was eating a gluten free diet, the child with Silver-Russell syndrome and a very short boy with Down's syndrome started growth hormone treatment, and the child found to be hypothyroid was given replacement thyroxine.

Although the mean height velocity of the normal short children was, as expected, significantly less than that of their controls $(p<0.01)$, there was considerable overlap of their distributions. Furthermore, whereas those with organic disease clearly ranked low for heigh when compared with normal short children, no such distinction could be made on the basis of velocity $(p=0.67)$. In those with newly diagnosed pathology, velocity $S D$ scores covered a similar range $(-2 \cdot 26$ to $0 \cdot 29$ ). There were no significant differences in mean velocity between the asthmatic and psychosocially deprived children and normal short children measured over 12 months $(p=0 \cdot 96 / p=0 \cdot 22)$.

\section{Discussion}

In this study of children at school entry we have shown, firstly, that short stature is an importan indicator of organic disease and, secondly, that height velocity, even over 12 months, does not distinguish individual short children with organic disease from normal short children. In the present study $18 \%$ of the children below the 3rd centile for height had organic disease which could have explained their short stature a figure comparable to the Newcastle study and not insubstantial. The proportion may be even higher, as 19 short children assumed to be normal failed to attend the outpatient clinic for further investigations. The finding of organic disease in more than one in four of those whose height lay more than $2.5 \mathrm{SD}$ below the mean is similar to the results of Vimpani et al - and in about half of those whose heights were more than 3 SD below the mean is similar to that reported by Lacey and Parkin. ${ }^{5}$ The increasing prevalence of organic disease with diminishing stature underpins the value of heigh as a screening test. We did not take blood samples from children above the 3 rd centile, so it is not possible to estimate the specificity of the test, but, in view of the trend we have observed, it can reasonably be assumed that only a small proportion would have had organic disease.

Height measurement is included in the medical examination at school entry with the aim of detecting underlying disease. Most organic disease accounting for short stature in the present study had been detected before school entry, but the school medical examination at school entry did not reveal the remaining organic disease in the short children. This remainder comprised one quarter of all cases and was treatable in about half.

Disease is not always evident, which explains why eight previously undiagnosed conditions were identified in this study. The children with coeliac disease and lead poisoning were eventually diagnosed after a low haemoglobin concentration was detected. The first had exceptionally short parents and had always been assumed to be short for genetic reasons. The second was of Asian origin and for that reason had never been considered unusually short. The two children with neurofibromatosis presented with café au lait spots and 
Scattergrams comparing mean and distribution of 12 month height velocities of control children (10th-90th centile for height), short normal children $(<3 r d$ centile for height $)$, and short children with organic disease

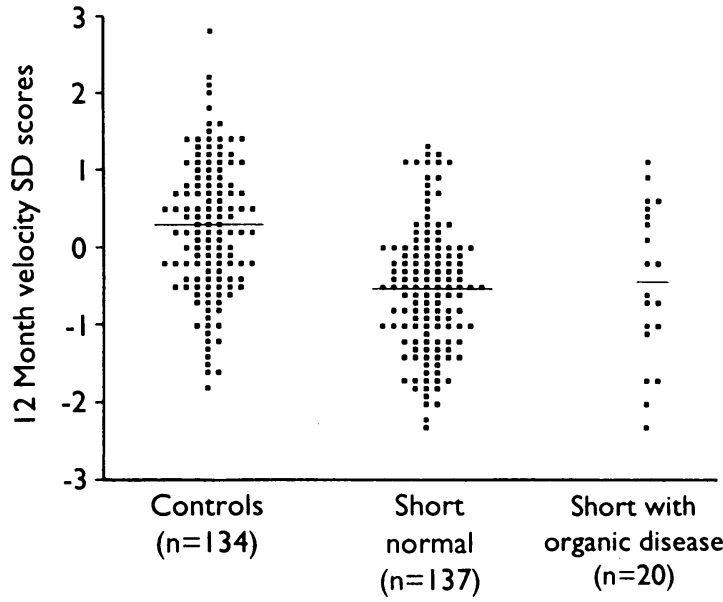

went on to have full skeletal surveys, as did the child with the trichorhinophalangeal syndrome, who was found to have abnormal radiographic features on a hand and wrist radiograph taken to estimate bone age. The child with acquired hypothyroidism was detected by a thyroid function test, and the growth hormone deficient child by a urinary growth hormone test. The former received immediate treatment and was there fore excluded from the 12 month velocity data.

Four children in our study had remediable conditions: hypothyroidism, coeliac disease, lead poisoning and growth hormone deficiency. This pick up rate ( 1 in $3000-4000$ ) may seem low as a percentage of the number of children initially screened for height but it is comparable to that for neonatal hypothyroidism ( 1 in 4300 ) and much higher than that for phenylketonuria ( 1 in 16000). ${ }^{13}$ In view of the serious nature of these disorders it may be argued that the routine investigation of very short children identified at school entry is worth while. We recommend the laboratory tests used in this study (full blood count, thyroid function test, and urinary growth hormone at least) for the shortest $\mathrm{x} \%$ of children at the school medical (the proportion depending on resources) and referral to the growth clinic of those found to have abnormalities. In view of the fact that the Tanner and Whitehouse standards used to screen for the shortest $3 \%$ of the school population nowadays identify as few as $1 \cdot 25 \%$ (because of secular trend ${ }^{8112}$ ) our proposals should not be too demanding.

Much has been made in the past of poor height velocity as a marker of organic disease, ${ }^{1+15}$ but its limitations in any screening programme have recently been made clear. ${ }^{10}$ There is considerable imprecision in the auxological assessment of growth. ${ }^{16}$ The error of measurement is so large that, although the expected difference in mean velocity between the short children and their controls was found, it was not possible to label any individual child as a good or poor grower after only 12 months of observation. From table I and the figure it is clear that normal short children and short children with organic disease have similar distributions of 12 month velocity, despite having significantly different distributions of height. This is in keeping with the low correlation between velocity and height. ${ }^{8}$

In the long term poor growth in children with organic disease would eventually be seen on the height chart, as in the case of children with Turner's syndrome, half of whom are still above the 3rd centile at the age of 5 , and also in acquired diseases such as hypothyroidism. For this reason the heights of all children should ideally be measured and plotted regularly as no child can be dismissed as normal without evidence of satisfactory growth. In practice, however height screening is used to identify those most likely to be at risk.

Although such screening has been widely promoted in the United Kingdom, ${ }^{17}$ the crucial step of what next to do with these short children, once identified, has never been resolved. As has been observed, ${ }^{18}$ a normal short child whose height lies on the 3 rd centile has an expected velocity on or about the 25 th centile. The recommendation until now in Wessex has been for school doctors to identify short children at school entry, to observe them for 12 months, and then to refer to a specialist all those who are growing "slowly." However, the use of the 25th centile for velocity as a threshold, as recommended by the Middlesex height velocity assessment chart, ${ }^{19}$ would have netted only $40 \%$ (8) of those with disease as well as $41 \%$ (56) of the short normal children.

We have shown that 12 month velocity measurements are not reproducible and therefore unlikely to be useful as a screening tool. ${ }^{10}$ Velocity cannot identify poor growth more quickly and does not improve upon the height data from which it is derived. Short stature, which is the integral of all past velocities, would seem to be a more immediate and therefore useful index of previously unrecognised organic disease.

\section{CONCLUSIONS AND RECOMMENDATIONS}

The measurement of height is a non-invasive screening tool which can help to identify disease. The shorter the stature the greater the likelihood of finding organic disease. Once the screening has been carried out on initial height, little is to be gained by waiting a year and screening on velocity. When already identified disease does not exclude a further cause of growth failure. No short child, whatever his or her parents' height or ethnic background, should be dismissed as norma without careful exclusion of underlying disease. The routine medical examination on school entry may fai to identify a substantial proportion of children with organic disease associated with short stature. Given short stature as the screening criterion, blood tests and experienced examination appear to be an essential next step.

LDV and JM are generously funded by Kabi Pharmacia and TJW by the Wellcome Trust. Our thanks to Mrs W Couper for preparing the manuscript.

1 Zerfas AJ. In: Falkner F, Tanner JM, eds. Human growth. 2nd ed. New York: Plenum, 1986:490.

2 Parkin JM. The short child. In: Brook CGD, ed. Clinical paediatric endocrinology. Oxford: Blackwell Scientific, 1989:96-117.

3 Kennedy FD. Have school entry medicals had their day? Arch Dis Child 1988;63:1261-3

4 Smith GC, Powell A, Reynolds K, Campbell CA. The five year school medical-time for change. Arch Dis Child 1990;65:225-7.

5 Lacey KA, Parkin JM. The causes of short stature: a community study of children in Newcastle on Tyne. Lancet 1974; ;:42-5.

6 Vimpani GV, Vimpani AF, Pocock SJ, Farquhar JW. Differences in physica characteristics, perinatal histories and social background between children with growth hormone deficiency and constitutional short stature. Arch $D$ Child 1981;56:922-8.

7 Cross AW. Health screening in schools. Part II. $\mathcal{F}$ Pediatr 1985;107:653-61

8 Voss LD, Walker J, Lunt H, Wilkin TJ, Betts PR. The Wessex growth study first report. Acta Paediatr Scand 1989;349(suppl):65-72.

9 Tanner JM, Whitehouse RH, Takaishi M. Standards from birth to maturity for height, weight, height velocity and weight velocity: British children 1965. Arch Dis Child 1966;41:454-71;613-5.

10 Voss LD, Wilkin TJ, Bailey BJR, Betts PR. The reliability of height and height velocity in the assessment of growth (the Wessex Growth Study) Arch Dis Child 1991:66:833-7.

11 Voss LD, Wilkin TJ, Betts PR. Do we need new growth charts? Lance 1987;ii:447-8.

12 Chinn S, Price CE, Rona RJ. Need for new reference curves for height. Arch Dis Child 1989;64:1545-53.

13 Donald RA, ed. Endocrine disorders. New York: Dekker, 1984

14 Tanner JM. Use and abuse of growth standards. In: Falkner F, Tanner JM eds. Human growth. 2nd ed. New York: Plenum, 1986:95-109.

15 Brook CGD, Hindmarsh PC, Smith PJ. Management of short stature. In Brook CGD, ed. Clinical paediatric endocrinology. Oxford: Blackwell Scientific Publications, 1989:1 18-27.

16 Voss LD, Bailey BJR, Cumming K, Wilkin TJ, Betts PR. The reliability of height measurement (the Wessex Growth Study). Arch Dis Child 1990;65: 1340-4

17 Aynsley-Green A, Macfarlane JA. Method for the earlier recognition of abnormal stature. Arch Dis Child 1983;58:535-7.

18 Hindmarsh PC, Brook CGD. Auxological and biochemical assessment of shor stature. Acta Paediatr Scand 1988;343(suppl):73-6.

19 Brook CGD, Hindmarsh PC. Tests for growth hormone secretion. Arch Dis Child 1991;66:85-7.

(Accepted 14 October 1992) 